\title{
The 1st EMBO workshop on PVC bacteria- Planctomycetes-Verrucomicrobia-Chlamydiae superphylum: Exceptions to the bacterial definition?
}

\author{
Damien P. Devos • Christian Jogler • \\ John A. Fuerst
}

Received: 17 May 2013/Accepted: 3 September 2013/Published online: 20 September 2013

(C) Springer Science+Business Media Dordrecht 2013

\begin{abstract}
The PVC superphylum is a phylogenetically supported collection of various related bacterial phyla that comprise unusual characteristics and traits. The 'PVC' abbreviation derives from Planctomycetes, Verrucomicrobia and Chlamydiae as members of this superphylum, while additional bacterial phyla are related. There has recently been increasing and exciting interest in the cell biology, physiology and ecology of members of this superphylum, including evolutionary implications of the complex cell organization of some species. It is timely that international
\end{abstract}

D. P. Devos $(\bowtie)$

Centre for Organismal Studies (COS),

Heidelberg University, Im Neuenheimer Feld 230,

69120 Heidelberg, Germany

e-mail: damien.devos@ cos.uni-heidelberg.de;

dpdevos@upo.es

Present Address:

D. P. Devos

Centro Andaluz de Biología del Desarrollo

(CSIC/UPO/JA), 41013, Sevilla, Spain

C. Jogler

Leibniz Institute, DSMZ (Deutsche Sammlung fur

Mikroorganismen und Zellkultur), Inhoffenstraße 7 B,

Braunschweig, Germany

e-mail: christian.Jogler@dsmz.de

\section{J. A. Fuerst}

School of Chemistry and Molecular Biosciences,

The University of Queensland, St. Lucia, Brisbane,

QLD 4072, Australia

e-mail: j.fuerst@uq.edu.au researchers in the PVC superphylum field met to discuss these developments. The first meeting entirely dedicated to those bacteria, the EMBO workshop "PVC superphylum: Exceptions to the bacterial definition" was held at the Heidelberg University to catalyze the formation of a vital scientific community supporting PVC-bacterial research. More than 45 investigators from more than 20 countries (PIs, senior scientists and students) attended the meeting and produced a great starting point for future collaborative research. This Special Issue will focus on the EMBOPVC meeting. This Perspective briefly summarizes the history of PVC-research, focusing on the key findings and provides a brief summary of the meeting with a focus on the major questions that arose during discussion and that might influence the research in the years to come.

Keywords Planctomycetes - Verrucomicrobia · Chlamydiae $\cdot$ Meeting

\section{PVC: what they are}

The initially surprising grouping of bacteria with such different characteristics as ability to infect eukaryotic cells intracellularly (in the case of members of the Chlamydiae) and ability to oxidize ammonia in wastewater anaerobically (in the case of anaerobic 
planctomycetes species) is now amply supported (Gupta et al. 2012; Hou et al. 2008; Kamneva et al. 2010; Pilhofer et al. 2008; Wagner and Horn 2006).

The PVC superphylum includes the phylum Planctomycetes, a group of ubiquitous bacteria comprising both aerobic and anaerobic representatives that dwell in soil, fresh water, the oceans, and most other locations in which microbial biodiversity has been analyzed (Fuerst and Sagulenko 2011); the phylum Verrucomicrobia, which includes a small number of species isolated from fresh water, soil and human feces and by implication the gastrointestinal tract (Derrien et al. 2004; Hedlund et al. 1997); the phylum Chlamydiae, whose members are obligate intracellular pathogens; the Poribacteria, found so far only as obligate symbiont of sponges (Fieseler et al. 2004) the phylum Lentisphaerae, which includes marine microorganisms and human gut microbiota (Cho et al. 2004) and the OP3 candidate phylum which contains no cultured representative but occurs in anoxic habitats (Glöckner et al. 2010). The inference that those organisms form a natural group was initially based on 16S rRNA phylogenetic analysis (Wagner and Horn 2006). However, since then it has been recovered by a range of further analyses and has become increasingly accepted as a valid taxonomic grouping (Gupta et al. 2012; Hou et al. 2008; Kamneva et al. 2010; Pilhofer et al. 2008; Pol et al. 2007; Fuerst 2013).

\section{Unusual traits}

These bacteria have characteristics that set them apart from other bacteria and in some aspects resemble eukaryotes or archaea more than other bacteria. For these reasons PVC superphylum members can be seen as exceptions within the domain Bacteria. These exceptional features include a complex and dynamic endomembrane system with no equivalent in prokaryotes, most clearly displayed in the phylum Planctomycetes but also in Verrucomicrobia and Lentisphaerae. It also includes many other features, such as tubulin in the case of some Verrucomicrobia; sterols in the case of the planctomycete Gemmata obscuriglobus; absence of peptidoglycan in planctomycetes (where it is replaced by protein in the cell wall); some verrucomicrobia and the chlamydiae (so far as has been determined); the lack in planctomycetes and chlamydiae of the otherwise ubiquitous FtsZ protein and FtsZ-dependent system for cell division widespread in all other bacteria; presence of $\mathrm{C} 1$ transfer enzymes in many planctomycetes; and the unique anaerobic ammonium-oxidizing metabolism in anammox planctomycetes (Fuerst and Sagulenko 2011).

The PVC are not immune to controversy, with different interpretations of the endomembrane organization, the exact relation of the cell wall type in planctomycetes to that of Gram-negative bacteria with their outer membranes, and the nature of the topology of the endomembranes which may envelop the nucleoid in some species, especially of the planctomycete genus G. obscuriglobus. These controversies have, in the wider evolutionary context, been related to the question of whether eukaryote-like features in the PVC superphylum are evolutionarily homologous or analogous to those of domain Eukarya and Archaea members (Devos 2012; Devos and Reynaud 2010; Forterre 2010; Forterre and Gribaldo 2010; Fuerst and Sagulenko 2011; McInerney et al. 2011; Reynaud and Devos 2011).

\section{Health- and industrial implications}

Various PVC member species have applied relevance to areas extending from human health to wastewater remediation and understanding the global cycling of nitrogen. Found in human gut, some verrucomicrobia (such as Akkermansia spp.) or some Lentisphaerae (such as Victivallis spp.) (van Passel et al. 2011) might prove relevant to human health and prevention of human disease, for example via the role of Akkermansia in mucin degradation in the intestine (Derrien et al. 2008; Everard et al. 2013; Png et al. 2010). The anammox species within the phylum Planctomycetes (such as Kuenenia stuttgartiensis) are of potential industrial interest, since their ability to oxidize ammonia to molecular nitrogen is the basis of patented industrial processes for wastewater remediation, reducing the excess nitrogen content of waste effluent, improving effluent quality from sewage without the need for aeration and thus saving energy, while sequestering carbon dioxide (van Dongen et al. 2001; Kartal et al. 2010). This process is also critical to operation of the global nitrogen cycle, producing much of the nitrogen in the air we breathe via anaerobic ammonium recycling in environments such as the ocean's oxygen minimum zones (Kalvelage 
et al. 2011). As a unique divergent unexplored group of bacteria, planctomycetes are an emerging source of novel secondary metabolites, which very well might be useful for drug development (Jeske et al. 2013).

\section{The $1^{\text {st }}$ meeting: EMBO workshop on PVC superphylum: Exceptions to the bacterial definition? Heidelberg, Germany}

The first meeting dedicated to these bacteria took place in early March 2013 at the Heidelberg University, thanks to a generous donation from the EMBO Courses and Workshops program (http://www.embo. org/funding-awards/courses-workshops). The small scale of the conference and of the community allowed for ample discussion between participants. This issue is a compilation of a selection of the talks. The full program can be found here: http://events.embo.org/ 13-pvc/. A short summary of the sessions follows:

Without any doubt the cell biology of planctomycetes is unique amongst bacteria and worth studying for new insights into the diversity of cell organization. However, while John Fuerst (The University of Queensland, Australia) in his keynote introductory talk confirmed his earlier published demonstration of a nucleoid-containing compartment in G. obscuriglobus that resembles the eukaryotic nucleus using cryosubstitution electron microscopy, freeze-fracture EM and reconstruction from serial sections as well as electron tomography, Rachel Santarella-Mellwig (EMBL, Germany) in collaboration with Damien Devos (Heidelberg University, Germany) presented a different view on G. obscuriglobus' cell biology emerging from thin sectioning and electron tomography that implies that such 'compartment' regions around the nucleoid may not be completely closed or surrounded by membranous envelopes (Santarella-Mellwig et al. 2013). While both findings appear mutually exclusive they might be explained either by different stages of the $G$. obscuriglobus life cycle (non-dividing versus dividing) or by the different techniques used. Future development of a synchronous division culture system may go towards resolving such apparent contradictions. Both talks highlighted that further studies are required to ultimately define the spatial compartmentalization in G. obscuriglobus during different stages in its life cycle. In addition novel artifact-free imaging techniques were demonstrated in the talk of Marga
Schüler (MPI, Germany). While technically still challenging such techniques also have high potential to ultimately answer this question in the future.

Consistent with compartmentalization in the aerobic heterotrophic G. obscuriglobus, Laura van Niftrik and Sarah Neumann (Radboud University of Nijmegen The Netherlands) presented results for the anaerobic autotrophic anammox planctomycetes K. stuttgartiensis from both electron tomography of sectioned cells and fractionation of whole cells into organelles. Reconstructions of membrane topology inside cells and immunogold labeling of ATP synthase are consistent with the concept of both paryphoplasm as a distinct region not to be confused with periplasm and a cytoplasmic membrane separate from the intracytoplasmic membrane and anammoxosome membrane. Sarah Neumann in Laura van Niftrik's group presented what may prove a major breakthrough in planctomycetes studies with her report of the successful fractionation of anammox cells consistent with the reality of the anammoxosome as a closed internal organelle and with the reality of the ribosome-containing and anammoxosome-containing pirellulosome as a separable compartment. Thus planctomycetes compartments seem to be confirmed by this approach, at least in anammox planctomycetes, but such approaches will need extension to other planctomycetes with similar elements of a shared cellular plan. These approaches will of course also prove very useful for study of the unique ammonium-oxidizing lithotrophic biochemistry of anammox planctomycetes.

Also consistent with compartmentalization of planctomycetes were the results of Ekaterina Yarunova from Naomi Ward's group (University of Wyoming, USA) suggesting that transcription and translation may well occur in separate compartments in G. obscuriglobus, an exciting result which if indeed valid would confirm earlier predictions made along these lines (Fuerst 2005).

However, consistent with the view that PVC bacteria may not be entirely exceptional in terms of compartmentalization was the study of Daan Speth in Mike Jetten's group (Radboud University of Nijmegen, The Netherlands) suggesting on the basis of genomic and bioinformatics analysis that planctomycetes and verrucomicrobia possess key components needed for generation of the typical Gram-negative outer membrane composition. Genes for outer-membrane proteins and their assembly and for LPS 
insertion were present in all genomes tested (Speth et al. 2012). In addition, the authors demonstrated that biomarkers of outer membrane biogenesis are highly expressed. The hypothesis of an outer membrane in planctomycetes at least will need rigorous experimental test, considering the highly proteinaceous composition already determined in past studies of this group (König et al. 1984), and consistent with another result at the Workshop, that of Muriel van Teeseling showing an S-layer type of lattice reminiscent of those in archaeal cell walls as a major cell wall component in the anammox $K$. stuttgartiensis.

More controversy about the compartmentalization of PVC bacteria as a whole was introduced by Martin Pilhofer (Caltech, USA) in his description of electron cryotomography of the verrucomicrobial genus Prosthecobacter, species of which produce the closest homologues to eukaryote tubulin known among bacteria (Pilhofer et al. 2011). Arguments were presented against the existence of the major planctomycetes cell plan compartments, the pirellulosome and the paryphoplasm. In addition, the intracytoplasmic nature of the membrane separating these compartments, known so far as the intracytoplasmic membrane, was questioned. However, Fuerst's group had earlier published results suggesting that the planctomycetes cell plan also applied to verrucomicrobia including Prosthecobacter (Lee et al. 2009). Thus, here is another debate requiring resolution by additional experiments and techniques. Relevant to the concept that many unusual PVC features may be eukaryote homologues, however, are the results of Martin Pilhofer confirming formation of microtubules by the bacterial tubulins of Prosthecobacter and their potential derivation from ancient rather than modern eukaryotic tubulins.

Relevant to potential eukaryotic homologues in planctomycetes were the intriguing results emerging from the study of the DNA damage response in planctomycetes, where unexpectedly homologous sequences were identified for ubiquitinases and kinases, signal transducers coupled to post-translational modification-controlled pathways in eukaryotes. In addition substantial differences in the kinome of planctomycetes relative to other bacteria were observed in the PVC genomes (Arcas et al. 2013).

The keynote talk from Michael Galperin (NIH, USA) on analysis of the evolution of bacterial membrane energetics in the PVC group and other Bacteria observed that the potentially ancestral mode of membrane energy conservation using the sodiummotive force has continued to be employed in various members of the PVC superphylum. Comparison of energy conservation in membranes of species in the three Domains of life indicated that PVC organisms are typical members of the Bacterial domain when judged from that perspective.

Ultimately, planctomycetes cell biology needs the tools of modern bacterial and eukaryote cell biology especially those of fluorescent proteins and protein fusions. This depends on development of genetic systems and Mareike Jogler of Christian Jogler's group (Leibniz Institute, DSMZ, Braunschweig, Germany) presented results indicating that, for the Planctomyces limnophilus model system, the use of GFP and translational fusions is close to being made useful as a tool for substantially accelerating the rate of progress in planctomycetes research. Comparative genomics by this group has also been a powerful tool for identifying putative genes involved in compartmentalization as well as cell division (Jogler et al. 2012), and we can now move on to spatial localization of expressed protein fusions based on these genes to illuminate their exact function. This will be even more enhanced by correlation with EM studies using, for example, focused ion beam milling combined with cryoelectron tomography, as described by Marga Schüler (MPI Biochemistry, Germany).

The occurrence of other compartments in members of the phylum Planctomycetes additional to those recognized as forming the shared planctomycetes cell plan, and occurrence of the genes involved in their formation, was suggested by the studies by Onur Ergilbin in Cheryl Kerfeld's group (UC Berkeley, USA) exploring the genomics and genetics of bacterial microcompartments in P. limnophilus. These microcompartments may be involved in utilization of diverse carbon sources, and may be important for our understanding of the planctomycetes ecology as well as their subcellular structure.

Relevant to both cell structure and ecology was the fascinating report of a thermophilic anaerobic planctomycete by Galina Slobodkina in Elizaveta BonchOsmolovskaya's group (Winogradsky Institute of Microbiology, Russia). As would be predicted from past studies of planctomycetes, compartmentalization by internal membranes was observed in these new pure planctomycete cultures isolated from a gold mine and a hot spring. 
As that study suggests, to fully understand the PVC group and such members as the planctomycetes we will need a full appreciation of their diversity. The studies from Olga Lage's group (Porto U., Portugal) on potential new species of Rhodopirellula and genera related to Rhodopirellula and Blastopirellula (Lage et al. 2013) and the studies of Jens Harder's group (MPI Marine Microbiology Bremen) also revealing new species of Rhodopirellula, point to the vast diversity which may remain to be explored even within existing genera and groups of genera within the single Planctomycetes phylum (Frank et al. 2013). Jens Harder's studies also revealed significant responses of Rhodopirellula strains to ammonium concentrations relating to the well known planctomycete phenomenon of rosette formation and their well established feature, one sometimes also frustrating to the experimenter, of aggregation in laboratory culture.

PVC superphylum members other than Planctomycetes have been less intensively explored regarding potential shared characteristics such as compartmentalization. However, compared to other bacteria such as Escherichia coli, even planctomycetes are barely studied at all. Comparative genomics is proving a powerful tool in strengthening the PVC knowledgebase, and Naomi Ward (U. Wyoming, USA) gave insights into how gene duplication and gene birth may have contributed to genome evolution in different PVC-groups, and suggested changes in co-translational secretion as a possible driver of novel endomembranes in these bacteria, with possible connections also with the sterol composition known to occur in the planctomycete G. obscuriglobus. Ilias Lagkouvardos from Matthias Horn's group (University of Vienna, Austria) described a small basic protein which forms a signature protein for members of the PVC superphylum, providing support for the validity of this group. A cell biology link is suggested by its DNA-binding ability (it is noted in passing that condensed nucleoids in cryosubstituted sectioned cells are a feature of many species of both the Planctomycetes and Verrucomicrobia phyla).

The phylum Verrucomicrobia is an important group of PVC bacteria widely distributed in soil, while some species such as Methylacidiphilum even inhabit extreme acid and high temperature habitats. Huub Op den Camp (Radboud University of Nijmegen) presented a masterful account of the complex physiology of these thermoacidophiles, growing at $\mathrm{pH}$ as low as 1 . Huub also outlined how carbon dioxide might be fixed by Methylacidiphilum fumariolicum and how carbon might be stored by this autotroph, with the role of glycogen storage described in detail, explaining some of the intriguing internal bodies found in these and perhaps other verrucomicrobia. Muriel van Teeseling reported about an ultrastructural study of a thermophilic and a mesophilic verrucomicrobial methanotroph. Both rod-shaped cells featured multiple electron light bodies (glycogen storage) and one or two electron dark bodies. Researchers from Nils-Kre Birkelånd's laboratory also presented results on the related species 'Methylacidiphilum kamchatkense' and the high conservation of their pmo genes encoding a methane monooxygenase. Since such methanotrophic verrucomicrobia can oxidize the potent greenhouse gas methane we need to know a lot more about them in their ecophysiological context.

The contributions from the chlamydiae scientific community were especially welcome at the Workshop, as we need to know considerably more about how the PVC supergroup membership might be relevant to features and evolution of this group of intracellular pathogens. Recently Matthias Horn (University of Vienna, Austria) demonstrated that chlamydiae species growing in protist (amoeba) cells such as the Parachlamydiacea, and Rhabchlamydiaceae constitute the most diverse families among the phylum Chlamydiae. Claire Bertelli from Gilbert Greub's laboratory (University of Lausanne, Switzerland) enlightened us concerning recent genomics for the chlamydia-related environmental bacteria or potential pathogens, with larger and more repeat elementcontaining genomes than animal pathogenic chlamydia, including Criblamydia and Estrella in the Criblamydiaceae family, as well as Protochlamydia. Studies from Matthias Horn's group demonstrate that comprehensive database analysis, including metagenomes, show that far from being confined to human or mammalian infection habitats, the Chlamydiae group is widespread and perhaps surprisingly includes marine habitats. Diversity in chlamydiae extends to the genome level, with transcriptional regulatory factors lacking conservation.

The developmental cycle of chlamydiae is relevant to the life cycle of PVC bacteria in general and their unusual features, as Lloyd Vaughan (University of Zurich) presented intriguing micrographs of 
potentially unusual cycles for the chlamydiae in members pathogenic for fish.

Historically, the origin of the interest in planctomycetes, and to some extent the verrucomicrobia within the PVC group, was their structure and morphology, and the production and interpretation of images has been central to their understanding. So it may well be suitable to end by highlighting Eveline Kolijn's view from an artist's and image-maker's perspective. Her talk on observation and visualization presented a fascinating story of the evolution of our use of the scientific image and how scientific imagery may be able to learn from the visual understanding of professional artists, as well as how the scientific image has had influence on the arts. In Eveline Kolijn's case this has included use of $P$. limnophilus cells in her technically advanced lithograph prints (Kolijn 2013). PVC bacteria are of course fascinating to the researchers studying them, and their reputation for elegance is already spreading to even wider audiences. One hopes that immense significance for evolution and ecology will also be appreciated much more widely as a result of this Workshop. Like a rosette of Planctomyces or Rhodopirellula, this Workshop will hopefully be just the first example of the value of aggregation and connecting for the PVC superphylum community. A second meeting is already in planning stages, and other methods for keeping in touch are also being organized actively (see the website www.pvcbacteria.org).

\section{References}

Arcas A et al (2013) Serine/threonine kinases and E2-ubiquitin conjugating enzymes in Planctomycetes: unexpected findings. Antonie van Leeuwenhoek. doi:10.1007/s10482013-9993-2

Cho J-C, Vergin KL, Morris RM, Giovannoni SJ (2004) Lentisphaera araneosa gen. nov., sp. nov, a transparent exopolymer producing marine bacterium, and the description of a novel bacterial phylum Lentisphaerae. Environ Microbiol 6:611-621. doi:10.1111/j.1462-2920.2004.00 614.x

Derrien M, Collado MC, Ben-Amor K et al (2008) The Mucin degrader Akkermansia muciniphila is an abundant resident of the human intestinal tract. Appl Environ Microbiol 74:1646-1648. doi:10.1128/AEM.01226-07

Derrien M, Vaughan EE, Plugge CM, de Vos WM (2004) Akkermansia muciniphila gen. nov., sp. nov., a human intestinal mucin-degrading bacterium. Int $\mathrm{J}$ Syst Evol Microbiol 54:1469-1476. doi:10.1099/ijs.0.02873-0
Devos DP (2012) Regarding the presence of membrane coat proteins in bacteria: Confusion? What confusion? BioEssays 34:38-39. doi:10.1002/bies.201100147

Devos DP, Reynaud EG (2010) Evolution. Intermediate steps. Science 330:1187-1188. doi:10.1126/science. 1196720

Everard A, Belzer C, Geurts L et al (2013) Cross-talk between Akkermansia muciniphila and intestinal epithelium controls diet-induced obesity. Proc Natl Acad Sci USA. doi:10. 1073/pnas.1219451110

Fieseler L, Horn M, Wagner M, Hentschel U (2004) Discovery of the novel candidate phylum "Poribacteria" in marine sponges. Appl Environ Microbiol 70:3724-3732. doi:10. 1128/AEM.70.6.3724-3732.2004

Forterre P (2010) A new fusion hypothesis for the origin of Eukarya: better than previous ones, but probably also wrong. Res Microbiol 162:77-91. doi:10.1016/j.resmic. 2010.10.005

Forterre P, Gribaldo S (2010) Bacteria with a eukaryotic touch: a glimpse of ancient evolution? Proc Natl Acad Sci USA 107:12739-12740. doi:10.1073/pnas.1007720107

Frank CS et al (2013) Genetic diversity of Rhodopirellula strains. Antonie van Leeuwenhoek. doi:10.1007/s10482013-0004-4

Fuerst JA (2005) Intracellular compartmentation in planctomycetes. Annu Rev Microbiol 59:299-328

Fuerst JA (2013) The PVC superphylum: exceptions to the bacterial definition? Antonie van Leeuwenhoek. doi:10. 1007/s10482-013-9986-1

Fuerst JA, Sagulenko E (2011) Beyond the bacterium: planctomycetes challenge our concepts of microbial structure and function. Nat Rev Microbiol 9:403-413. doi:10.1038/ nrmicro 2578

Glöckner J, Kube M, Shrestha PM et al (2010) Phylogenetic diversity and metagenomics of candidate division OP3. Environ Microbiol 12:1218-1229. doi:10.1111/j.14622920.2010.02164.x

Gupta RS, Bhandari V, Naushad HS (2012) Molecular signatures for the PVC clade (Planctomycetes, Verrucomicrobia, Chlamydiae, and Lentisphaerae) of bacteria provide insights into their evolutionary relationships. Front Evol Genomic Microbiol 3:327. doi:10.3389/fmicb.2012.00327

Hedlund BP, Gosink JJ, Staley JT (1997) Verrucomicrobia div. nov., a new division of the bacteria containing three new species of Prosthecobacter. Antonie Van Leeuwenhoek 72:29-38

Hou S, Makarova KS, Saw JHW et al (2008) Complete genome sequence of the extremely acidophilic methanotroph isolate V4, Methylacidiphilum infernorum, a representative of the bacterial phylum Verrucomicrobia. Biol Direct 3:26. doi:10.1186/1745-6150-3-26

Jeske O et al (2013) From genome mining to phenotypic microarrays: Planctomycetes as source for novel bioactive molecules. Antonie van Leeuwenhoek. doi:10.1007/ s10482-013-0007-1

Jogler C, Waldmann J, Huang X et al (2012) Identification of proteins likely to be involved in morphogenesis, cell division, and signal transduction in planctomycetes by comparative genomics. J Bacteriol 194:6419-6430. doi:10. 1128/JB.01325-12

Kalvelage T, Jensen MM, Contreras S et al (2011) Oxygen sensitivity of anammox and coupled $\mathrm{N}$-cycle processes in 
oxygen minimum zones. PLoS ONE 6:e29299. doi:10. 1371/journal.pone.0029299

Kamneva OK, Liberles DA, Ward NL (2010) Genome-wide influence of indel Substitutions on evolution of bacteria of the PVC superphylum, revealed using a novel computational method. Genome Biol Evol 2:870-886. doi:10.1093/ gbe/evq071

Kartal B, Kuenen JG, van Loosdrecht MCM (2010) Engineering. Sewage treatment with anammox. Science 328:702703. doi:10.1126/science. 1185941

Kolijn E (2013) Observation and visualization: reflections on the relationship between science, visual arts, and the evolution of the scientific image. Antonie van Leeuwenhoek. doi:10.1007/s10482-013-9951-z

König E, Schlesner H, Hirsch P (1984) Cell wall studies on budding bacteria of the Planctomyces/Pasteuria group and on a Prosthecomicrobium sp. Arch Microbiol 138:200205. doi: $10.1007 / \mathrm{BF} 00402120$

Lage OM et al (2013) Insights into the ultrastructural morphology of novel Planctomycetes. Antonie van Leeuwenhoek. doi:10.1007/s10482-013-9969-2

Lee K-C, Webb R, Janssen P et al (2009) Phylum Verrucomicrobia representatives share a compartmentalized cell plan with members of bacterial phylum Planctomycetes. BMC Microbiol 9:5. doi:10.1186/1471-2180-9-5

McInerney JO, Martin WF, Koonin EV et al (2011) Planctomycetes and eukaryotes: a case of analogy not homology. BioEssays 33:810-817. doi:10.1002/bies.201100045

Pilhofer M, Ladinsky MS, McDowall AW et al (2011) Microtubules in bacteria: ancient tubulins build a five-protofilament homolog of the eukaryotic cytoskeleton. PLoS Biol 9:e1001213. doi:10.1371/journal.pbio.1001213

Pilhofer M, Rappl K, Eckl C et al (2008) Characterization and evolution of cell division and cell wall synthesis genes in the bacterial phyla Verrucomicrobia, Lentisphaerae,
Chlamydiae, and Planctomycetes and phylogenetic comparison with rRNA genes. J Bacteriol 190:3192-3202. doi:PMC2347405

Png CW, Lindén SK, Gilshenan KS et al (2010) Mucolytic bacteria with increased prevalence in IBD mucosa augment in vitro utilization of mucin by other bacteria. Am J Gastroenterol 105:2420-2428. doi:10.1038/ajg.2010.281

Pol A, Heijmans K, Harhangi HR et al (2007) Methanotrophy below $\mathrm{pH} 1$ by a new Verrucomicrobia species. Nature 450:874-878. doi:10.1038/nature06222

Reynaud EG, Devos DP (2011) Transitional forms between the three domains of life and evolutionary implications. Proc Biol Sci 278:3321-3328. doi:10.1098/rspb.2011.1581

Santarella-Mellwig R, Pruggnaller S, Roos N et al (2013) Threedimensional reconstruction of bacteria with a complex endomembrane system. PLoS Biol 11:e1001565. doi:10. 1371/journal.pbio.1001565

Speth DR, van Teeseling MCF, Jetten MSM (2012) Genomic analysis indicates the presence of an asymmetric bilayer outer membrane in Planctomycetes and Verrucomicrobia. Front Evol Genomic Microbiol 3:304. doi:10.3389/fmicb. 2012.00304

Van Dongen U, Jetten MS, van Loosdrecht MC (2001) The SHARON-Anammox process for treatment of ammonium rich wastewater. Water Sci Technol J Int Assoc Water Pollut Res 44:153-160

Van Passel MWJ, Kant R, Palva A et al (2011) Genome sequence of Victivallis vadensis ATCC BAA-548, an anaerobic bacterium from the phylum Lentisphaerae, isolated from the human gastrointestinal tract. J Bacteriol 193:2373-2374. doi:10.1128/JB.00271-11

Wagner M, Horn M (2006) The Planctomycetes, Verrucomicrobia, Chlamydiae and sister phyla comprise a superphylum with biotechnological and medical relevance. Curr Opin Biotechnol 17:241-249 